\title{
基于三脚架配体构筑的钣系-配体多重键的研究进展
}

\author{
李斌 $\uparrow, a$ 于吉攀 $\uparrow, b$ 刘康 $b \quad$ 吴群燕 $b$ 刘琦*, $a$ 石伟群 $*, b$ \\ ( ${ }^{a}$ 哈尔滨工程大学材料科学与化学工程学院 哈尔滨 150001) \\ ( $b$ 中国科学院高能物理研究所 北京 100049)
}

\begin{abstract}
摘要 非水溶液体系的铜系元素化学是一个极具挑战性的前沿研究领域, 近年来在分子磁性、多重键以及小分子活化 等方面获得了迅速发展. 化学键是化学科学中最重要的基本概念之一, 而金属配体多重键是该领域重要的研究内容. 多重键的形成与钧系元素的电子结构密切相关, 相对论效应使得铜系元素的 $\mathrm{s}$ 轨道和 $\mathrm{p}$ 轨道收缩, 轨道能量降低, 收缩 的 $\mathrm{s}$ 和 $\mathrm{p}$ 轨道增加了对核电荷的屏蔽效应, 从而使 $\mathrm{d}$ 和 $\mathrm{f}$ 轨道具有一定的延展性和不稳定性. 这种不稳定性降低了 $5 \mathrm{f}$ 电子的结合能, 电子更容易离去, 可使铜系元素具有丰富的氧化态. 由于较高的主量子数和相对论效应, 铜系元素的 $5 \mathrm{f}$ 轨道具有更大的径向延展, 在铜系元素中 $5 \mathrm{f}$ 轨道的电子行为影响较大. 目前, 铜系金属-配体多重键因其独特的成键 方式和电子结构特征而受到科学家的广泛关注, 在合成和分离方面存在极大的挑战, 研究铜系-配体多重键将有助于 我们了解它们的电子结构和反应性. 基于口袋型拓扑结构的三脚架配体被广泛地应用于钣系-配体多重键的研究, 这 为探索铜系元素的 $5 \mathrm{f}$ 电子结构和钶系多重键丰富的化学行为提供了重要支撑. 本综述总结了近年来基于三脚架配体 构筑的铜系-配体多重键的研究进展, 并对未来进行了展望.
\end{abstract}

关键词＼cjkstart铜系元素; 三脚架配体; 配合物; 铜系-配体多重键; 小分子活化

\section{Research Progress of Actinide-Ligand Multiple Bonding Supported by Tripodal Ligands}

\author{
Bin $\mathrm{Li}^{\dagger, a} \quad$ Jipan $\mathrm{Yu}^{\dagger, b} \quad \mathrm{Kang} \mathrm{Liu}^{b} \quad{\text { Qunyan } \mathrm{Wu}^{b} \quad \text { Qi Liu*,a }}^{*}$ Weiqun Shi*,b \\ ( ${ }^{a}$ College of Materials Science and Chemical Engineering, Harbin Engineering University, Harbin 150001, China) \\ ( ${ }^{b}$ Institute of High Energy Physics, Chinese Academy of Sciences, Beijing 100049, China)
}

\begin{abstract}
Nonaqueous actinide chemistry is a challenging frontier field, which has made rapid progress in molecular magnetism, multiple bonds and small molecular activation in recent years. Chemical bond is an important basic concept in chemical science, and metal-ligand multiple bond becomes important research content in this field. The formation of multiple bonds is closely related to the electronic configuration of actinides. The s and p-orbitals of actinides contract toward the nucleus and thus produce lower energy level, which increase the shielding effect on nuclear charge. This leads to indirect relativistic effects where the $\mathrm{d}$ and f-orbitals experience expansion and destabilization. This destabilization decreases the binding energy of $5 \mathrm{f}$ electrons and make them more easily to leave, giving rise to a large range of available oxidation states. Due to higher principle quantum number and relativistic effects, the $5 \mathrm{f}$ orbitals of actinides have a much greater radial extension, and the electronic effect of $5 \mathrm{f}$ orbitals has a greater influence. At present, the research of actinide-ligand multiple bonds has become one of the most remarkable fields in actinide chemistry and received extensive attention. It is a great challenge to synthesize and separate compounds with actinide-ligand multiple bonds due to their strong radioactivity and toxicity and complex electronic structures. To study actinide-ligand multiple bonds, especially double- and triple-bonded complexes, will assist us to understand their electronic structure, reactivity and physical properties such as electrical conductivity, magnetism, and photochemistry. Tripodal ligands with pocket topology have been widely used in the research of actinide-ligand multiple bonds. This gives a window into exploring the diverse chemical behavior of actinide multiple bonds and observing how actinides can utilize its $5 \mathrm{f}$ electrons to exhibit interesting and novel chemistry. The research progress of actinide-ligand multiple bonds based on tripodal ligand is summarized in this review, and the prospect of this field is prognosticated.
\end{abstract}

Keywords actinide element; tripodal ligand; coordination complex; actinide-ligand multiple bond; small molecule activation

\footnotetext{
* E-mail: qiliu@hrbeu.edu.cn; shiwq@ihep.ac.cn

$\uparrow$ These two authors contributed equally to this work.

Received April 9, 2021; published May 25, 2021.

Project supported by the National Natural Science Foundation of China (Nos. 21925603, 21806167).

国家自然科学基金项目(Nos. 21925603, 21806167)资助.
} 


\section{1 引言}

钣系金属有机化学是研究钣系元素成键特性和理 化性质的重要领域, 因其研究门槛高, 技术难度大, 世 界上只有为数不多的课题组在开展相关工作, 而且相关 理论研究工作也非常贵乏. 近年来, 钶系-配体多重 键 ${ }^{[1-2]}$ 引起了人们极大的兴趣, 其相关研究对于理解钣可 系配合物的电子结构和成键性质至关重要, 而且有助于 人们更加深刻地理解 $5 \mathrm{f}$ 和/或 $6 \mathrm{~d}$ 轨道参与的成键特征, 为乏燃料后处理中钣系元素的分离提供科学依据和理 论指导.

配体的选择对于含有钶系-配体多重键配合物的合 成至关重要. 由于钣系元素配位数普遍较高, 选用多齿 配体在钣系-配体多重键的研究中非常重要. 例如: bisiminophosphoranomethanediide $\left\{\mathrm{C}\left(\mathrm{PPh}_{2} \mathrm{NR}\right)_{2}\right\}^{2-}\left(\mathrm{BIPM}^{\mathrm{R}}\right)$ 作为一种三齿的 Pincer 型卡宾配体构筑了多种结构类型 的 $\mathrm{An}=\mathrm{C}$ 双重键的配合物 ${ }^{[3-5]}$. 而具有三脚架结构的四 齿配体因其独特的结构在钣系-配体多重键的研究中获 得广泛应用, 它具有以下特点: (1)特殊的结构促使配位 的金属中心具有单一反应位点; (2)骨架上的取代基可以 有效调控其位阻大小和反应活性来实现特定的反应; (3) 提供了一个口袋型的骨架来螯合高配位数的钶系元素, 这种饱和配位避免了反应过程中配合物的分解. 因此, 三脚架配体可以调控钶系配合物的稳定性、兼容性和反 应性. Tren、Tacn 和 TriNOx 都为三脚架型的四齿配体, 图 1 为配体的合成方法. 本综述针对以上三类三脚架型 配体构筑的钣系-配体多重键的工作进行详细的归纳和 总结.

\section{2 基于 Tren 配体的钣系-配体多重键}

三(2-胺基乙基)胺(简称为 Tren)配体是一类重要的 多齿配体, 其在材料、萃取剂和均相催化剂等方面已经 获得了广泛的应用. 由于其特殊的口袋型构象, 各种官 能团取代的 Tren 配体可以很好地螯合钣可系元素，因而 在钣系-配体多重键配合物的合成中具有潜在的优势. 如图 2 所示, 三异丙基硅基保护的 Tren 配体可以分别与 $\mathrm{UCl}_{4}{ }^{[6]}$ 和 $\mathrm{ThCl}_{4}{ }^{[7]}$ 反应，构筑 $\mathrm{U}\left(\operatorname{Tren}^{\mathrm{TIPS}}\right) \mathrm{Cl}$ 和 $\mathrm{Th}\left(\operatorname{Tren}^{\mathrm{TIPS}}\right) \mathrm{Cl}$ 这两种重要的前体化合物，该前体化合物 可以通过各种反应实现其衍生化, 也为后续多重键的构 筑奠定了基础.

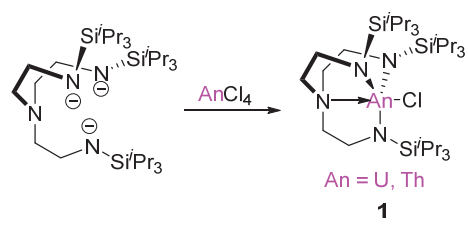

图 2 Tren 配体螯合铀和针的前体化合物的合成

Figure 2 Syntheses of uranium and thorium complexes supported by tren ligand

2012 年, Liddle 课题组 ${ }^{[6]}$ 采用三异丙基硅基保护的 Tren 配体螯合的三价铀配合物 2 和叠氮化钠反应，通过 两电子的氧化, 生成了钠桥联的 $\mathrm{U} \equiv \mathrm{N}$ 三重键的配合物 3(图 3). 晶体结构显示 $U-N$ 的键长为 0.1883 (4) $\mathrm{nm}$ (化 合物 3), 验证了 $\mathrm{U} \equiv \mathrm{N}$ 三重键的存在, 这也是首例含有 $\mathrm{U} \equiv \mathrm{N}$ 三重键的单晶结构. 加入 12-冠-4 用于络合钠离 子, 得到了电荷分离的 $\mathrm{U} \equiv \mathrm{N}$ 三重键的配合物 4. 晶体

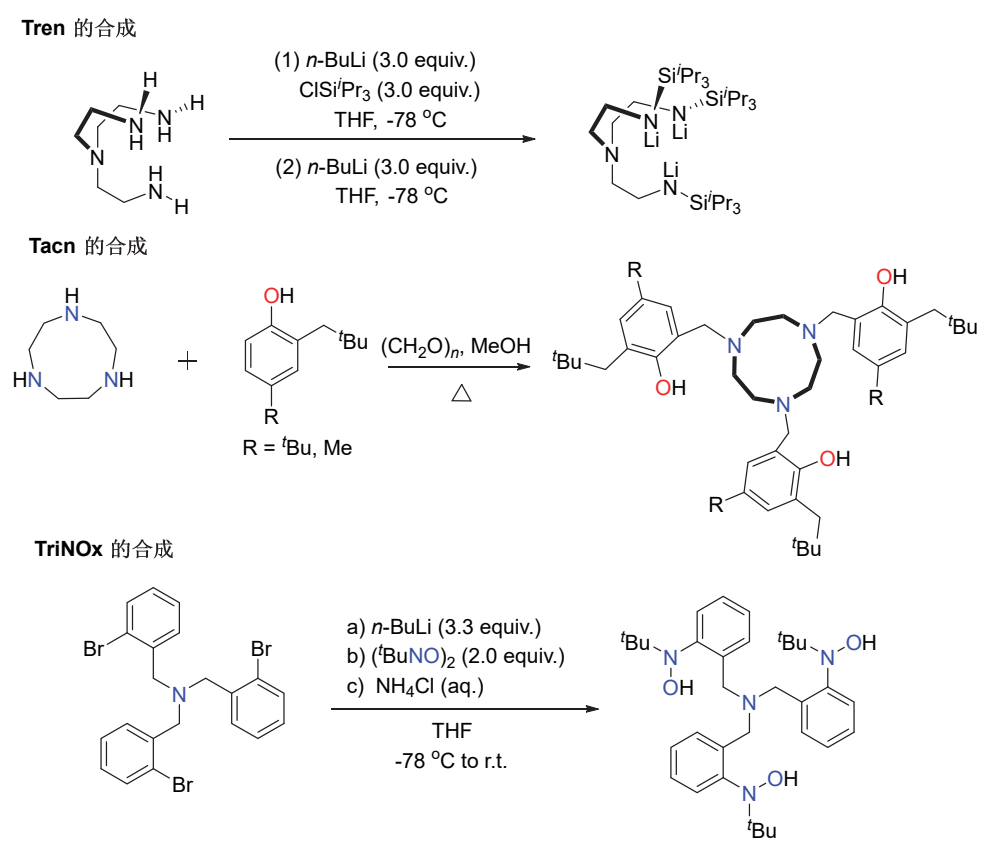

图 1 配体 Tren、Tacn 和 TriNOx 的合成

Figure 1 Syntheses of Tren, Tacn and TriNOx ligands 


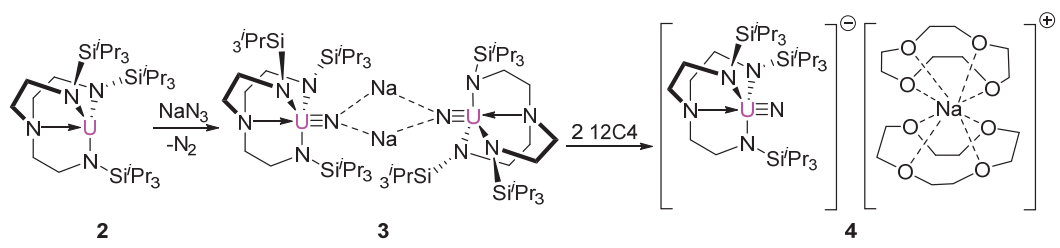

图 3 Tren 配体螯合的 $\mathrm{U}(\mathrm{V}) \equiv \mathrm{N}$ 三重键配合物的合成

Figure 3 Synthesis of $\mathrm{U}(\mathrm{V}) \equiv \mathrm{N}$ triple bond complex supported by tren ligand

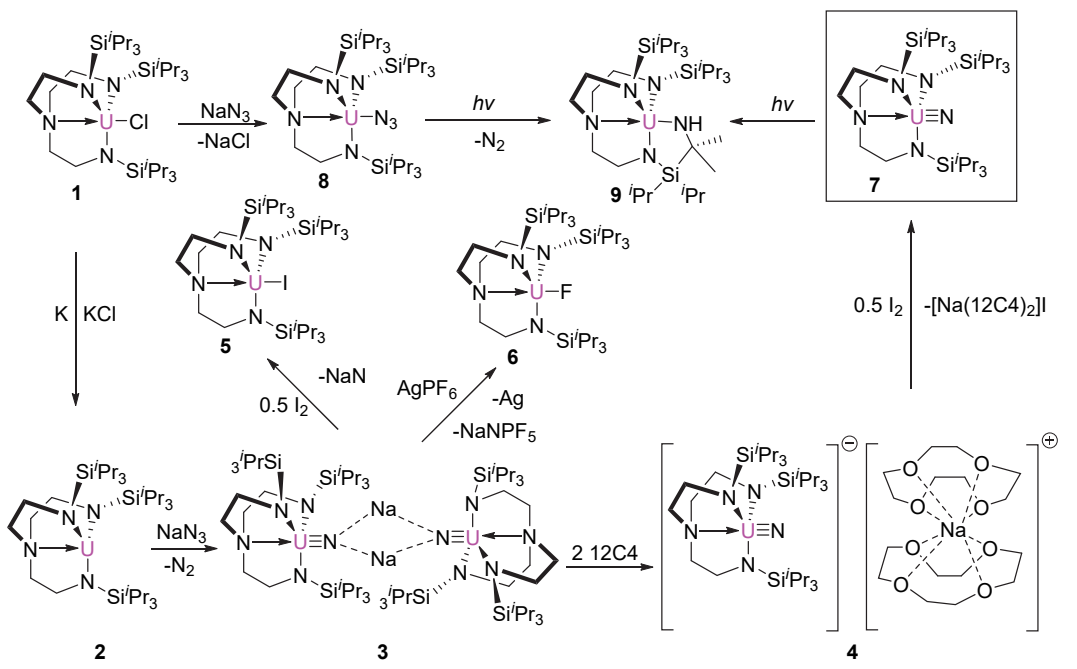

图 $4 \mathrm{U}(\mathrm{VI}) \equiv \mathrm{N}$ 三重键配合物的合成

Figure 4 Syntheses of $\mathrm{U}(\mathrm{VI}) \equiv \mathrm{N}$ triple bond complexes

结构显示 $U-\mathrm{N}$ 的键长为 $0.1825(15) \mathrm{nm}$ (化合物 4). 以上 两个均是含有 $U \equiv N$ 三重键的五价铀配合物. 而电荷分 离的配合物 4 中的 $\mathrm{U} \equiv \mathrm{N}$ 三重键的键长比配合物 3 中的 键长略短.

随后，作者进一步对化合物 4 采用分子碘氧化的方 式 ${ }^{[8]}$, 首次合成得到了末端 $\mathrm{U}(\mathrm{VI}) \equiv \mathrm{N}$ 三重键的中性配 合物 7(图 4). 然而化合物 3 的氧化并没有得到六价铀配 合物，而是生成了四价铀的碘代物 5 和氟代物 6 . 而且 7 可以在光照条件下通过分子内的 $\mathrm{C}-\mathrm{H}$ 键活化得到化合 物 9, 并生成新的 $\mathrm{N}-\mathrm{H}$ 键和 $\mathrm{C}-\mathrm{N}$ 键; 同样, $\mathrm{U}-\mathrm{N}_{3}$ 配合 物 8 在光照条件也得到化合物 $\mathbf{9}$, 这说明 U-N 3 配合物 8 在光照条件下首先生成了 $\mathrm{U}(\mathrm{VI}) \equiv \mathrm{N}$ 的配合物 $7[\mathrm{U}-\mathrm{N}$ 的键长为 0.1799(7) $\mathrm{nm}$ ], 并很快地转化为 9. 该结果和 Kiplinger 课题组 ${ }^{[9]}$ 在 2010 年发现的实验现象非常相似, 也进一步验证了铀-氮三重键中间体的生成. 而配合物 7 中的 $\mathrm{U} \equiv \mathrm{N}$ 三重键的键长比配合物 $\mathbf{4}$ 中的键长略短.

2013 年, Liddle 课题组 ${ }^{[10]}$ 研究发现三价的 $\mathrm{U}\left(\operatorname{Tren}^{\mathrm{TIPS}}\right)$ 配合物 2 可被氮氧化物氧化为五价的 $\mathrm{U}=\mathrm{O}$ 配合物 10, 晶体结构显示 $U-O$ 的键长为 $0.1856(6) \mathrm{nm}$ (图 5). 而且 10 表现出单分子磁体 (SMM, singlemolecule magnets)的行为, 这是第一个单金属五价铀的 单分子磁体, 也是首个单金属含有 $\mathrm{f}^{1}$ 电子构型的单分子 磁体. 其结果表明, 五价铀的配合物是目前最有希望获 得 $5 \mathrm{f}$ 构型的单分子磁体. 由于 Tren 配体具有强的轴向
配位以及可协调的周围配位环境，五价的铀氧双键配合 物的对称性可以被有效地控制. 因此, 末端含有 $U(V)=$ $\mathrm{O}$ 双重键的配合物将会在单分子磁体领域发挥重要的 作用.
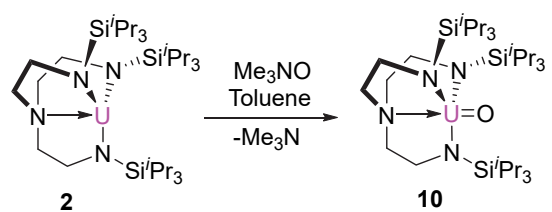

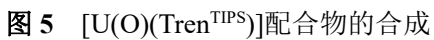

Figure 5 Synthesis of $\left[\mathrm{U}(\mathrm{O})\left(\operatorname{Tren}^{\mathrm{TIPS}}\right)\right]$ complex

2014 年, Liddle 课题组 ${ }^{[11]}$ 对 $U \equiv \mathrm{N}$ 三重键配合物的 化学性质进行了详细的研究. 如图 6 所示, 研究结果表 明 $\mathrm{U} \equiv \mathrm{N}$ 配合物 7 通过 $\mathrm{KC}_{8}$ 还原可以得到钾离子桥联的 $\mathrm{U} \equiv \mathrm{N}$ 配合物 11, 此化合物进一步被冠醚螯合得到电荷 分离的配合物 12,12 可与 $\mathrm{CO}$ 通过两电子还原反应得到 三价铀的异氰酸酷配合物 13,13 可通过分子碘氧化得到 四价铀的异氧酸酷配合物 14. 重要的是, $U \equiv N$ 配合物 7 具有较高的反应活性, 可以直接和 $\mathrm{CO}$ 通过两电子还原 的方式得到四价铀的异氧酸酯配合物 $\mathbf{1 4 , 1 4}$ 可通过 $\mathrm{KC}_{8}$ 还原并采用冠醚鳌合钾离子的方式重新生成三价铀的 异氧酸酯配合物 13. 所以, 通过氧化还原反应可以实现 各种价态铀之间的相互转化, 同时也说明三价铀前体和 $\mathrm{U} \equiv \mathrm{N}$ 配合物具有较高的反应活性. 


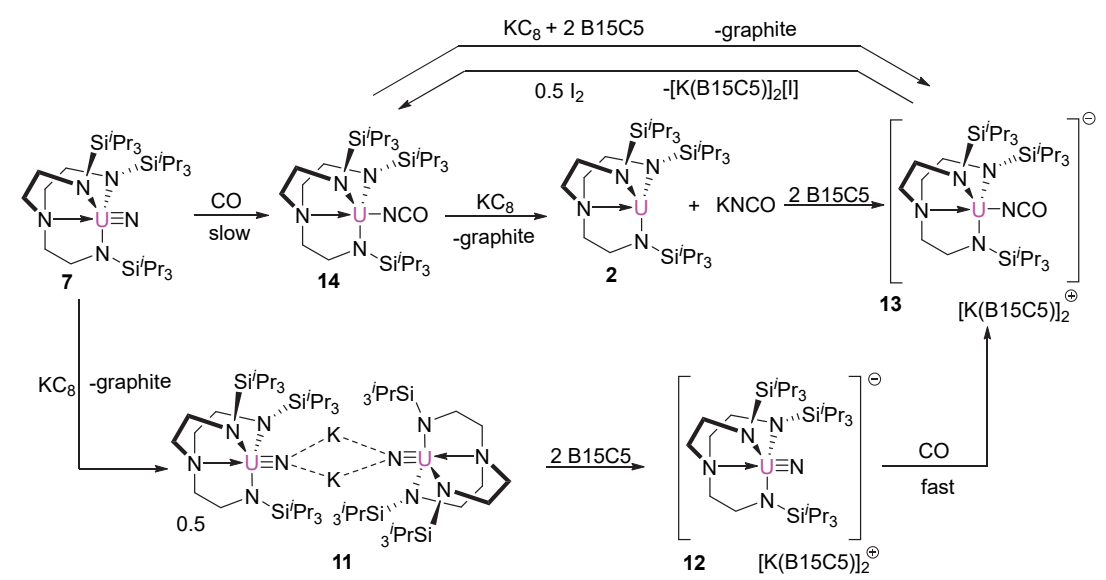

图 6 铀氮三重键配合物的反应性研究

Figure 6 Study the reactivity of uranium-nitride complex

2014 年, Liddle 课题组 ${ }^{[12]}$ 以 U(IV)- $\mathrm{NH}_{2}$ 为底物通过 碱金属脱质子得到了含有亚氨基的双核铀配合物 16 $[\mathrm{U}-\mathrm{N}$ 的键长范围为 $0.2042(3) \sim 0.2135(3) \mathrm{nm}], 16$ 中的 碱金属离子可被穴醚包合得到电荷分离的配合物 17 (图 7). 化合物 $\mathbf{1 7}[\mathrm{U}-\mathrm{N}$ 的键长为 0.2034(3) nm] 被视为一个 隐蔽的铀(IV)-氮化物, 而且铀-亚胺键直线型的连接方 式具有 $\sigma^{2} \pi^{4}$ 电子构型的 $U-N$ 极化铀氮多重键. 17 进一 步通过分子硔氧化得到末端含有 $U(V I) \equiv N$ 三重键的中
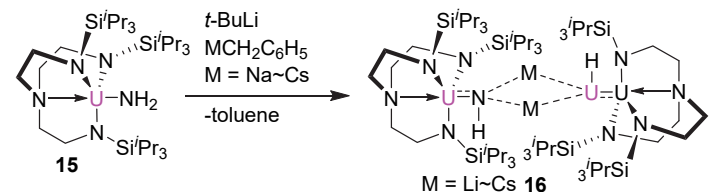

$\mathrm{M}=\mathrm{Li} \sim \mathrm{Cs} 16 \quad{ }_{3} \mathrm{Pr}$

$$
M=K \mid 215 \mathrm{C} 5
$$

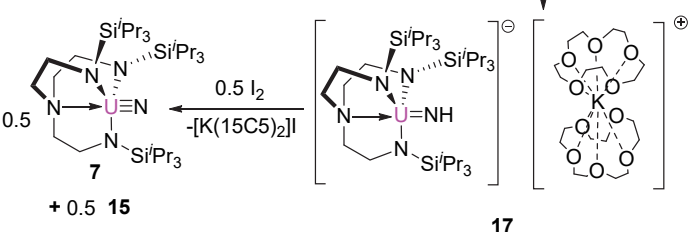

图 7 铀的亚氨基配合物和铀-氮三重键配合物的合成

Figure 7 Syntheses of uranium-imido and uranium-nitride complexes
性配合物 7 以及 $\mathrm{U}(\mathrm{IV})-\mathrm{NH}_{2}$ 化合物 $\mathbf{1 5}$. 以上内容是合成 铀氮三重键的新策略，也为其他铜系多重键配合物的合 成提供了新思路.

由于电子间固有的排斥、晶体场效应和旋轨耦合效 应的影响，确定钣系配合物的电子结构仍然具有很大的 挑战. 如图 8 所示, 2016 年, Liddle 课题组 ${ }^{[13]}$ 采用 $\mathrm{Li} \sim \mathrm{Cs}$ 五种碱金属还原 U(IV)- $\mathrm{N}_{3}$ 配合物 8, 成功合成了金属桥 联的 $\mathrm{U}(\mathrm{V}) \equiv \mathrm{N}$ 配合物 $18[\mathrm{U}-\mathrm{N}$ 的键长范围为 $0.1846(3) \sim 0.1929(6) \mathrm{nm}$ ]. 在 $\mathbf{1 8}$ 中加入 1 equiv.不同类 型的冠醚可以很好地螯合碱金属离子, 形成 $\mathrm{U} \equiv \mathrm{N}-\mathrm{M}$ 构型的配合物 19 [U-N 的键长范围为 0.1801(7) $0.1825(15) \mathrm{nm}]$; 加入 2 equiv. 不同类型的冠醚可以形成 电荷分离的配合物 20 [U-N 的键长范围为 $0.1803(5) \sim$ $0.1840(3) \mathrm{nm}$. 以上碱金属鳌合的配合物中铀的价态都 为 +5 价, 和 $\mathrm{U}(\mathrm{V})=\mathrm{O}$ 是等电子体, 可以采用磁性测量、 电子顺磁共振(EPR)和各种光学手段结合建模的方法来 确定其电子结构, 并通过明确自旋轨道和晶体场相互作 用的重要性，有效地解释了 $\mathrm{N}^{3-}$ 配合物比 $\mathrm{O}^{2-}$ 配合物具 有更加复杂的基态电子结构. 此外, 通过探索整个系列 配合物的磁性，证明它们为单分子磁体.

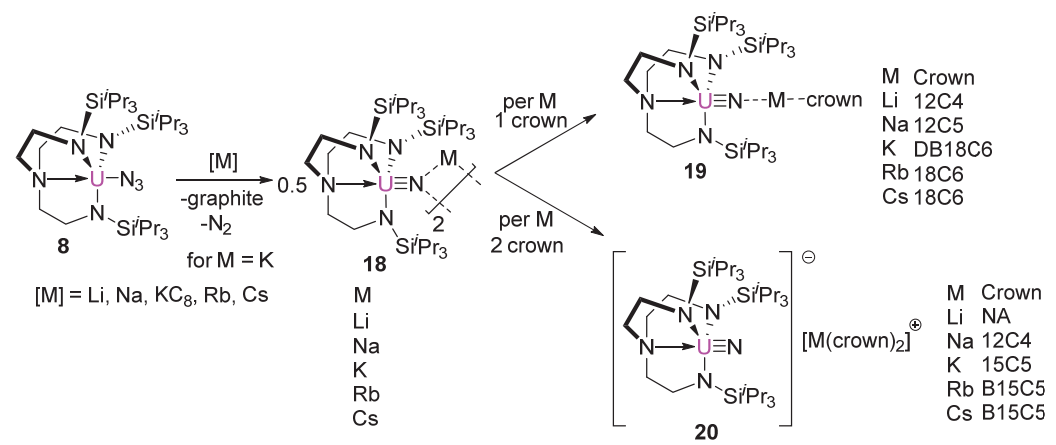

图 8 碱金属还原 $\mathrm{U}(\mathrm{IV})-\mathrm{N}_{3}$ 配合物合成 $\mathrm{U} \equiv \mathrm{N}$ 三重键配合物

Figure 8 Syntheses of uranium nitride complexes by reduction of U(IV)- $\mathrm{N}_{3}$ using alkali metal 
2017 年, Liddle 课题组 ${ }^{[14]}$ 进一步开展了 U(V/VI) $\equiv \mathrm{N}$ 配合物与 $\mathrm{CO}_{2}$ 和 $\mathrm{CS}_{2}$ 等小分子反应性的研究(图 9). 研究 发现, $\mathrm{U}(\mathrm{V}) \equiv \mathrm{N}$ 配合物 12 和 $\mathrm{CS}_{2}$ 反应可以促进 12 的歧 化, 得到六价铀的氮化物 7 和四价铀的三硫碳酸酯衍生 物 21. 12 和 $\mathrm{CO}_{2}$ 反应得到具有 $\mathrm{U}(\mathrm{V})=\mathrm{O}$ 结构单元的异 㲵酸酯类衍生物 22 [U-O 的键长为 $0.1848(7) \mathrm{nm}$ ]. 而 $\mathrm{U}(\mathrm{VI}) \equiv \mathrm{N}$ 配合物 7 和 $\mathrm{CS}_{2}$ 反应与上述反应有所异同, 得 到异硫氰酸酯的四价铀配合物 23, 并得到单质硫, 这说 明 $\mathrm{CS}_{2}$ 作为还原剂实现了 $\mathrm{U}(\mathrm{VI})$ 到 $\mathrm{U}(\mathrm{IV})$ 的转化. U(VI) $\equiv \mathrm{N}$ 配合物 7 和 $\mathrm{CO}_{2}$ 反应很快会形成红色的配合物 $\mathbf{2 4}$, 而 24 不稳定, 会分解为 $U(V)=O$ 配合物 10 . 产物 10 的 产生表明有异氰酸酯自由基从配合物 24 上脱除并形成 二聚体的形式，并进一步分解为 $\mathrm{N}_{2}$ 和 CO.

由氮气和氢气合成氨的非均相 Haber-Bosch 反应中 机理模型很重要; 而均相反应中金属氮化物与氢气的反 应一般认为是惰性的, 只有少数富电子、低配位的配合 物可以发生氢化反应. 2020 年, Liddle 课题组 ${ }^{[15]}$ 研究发 现缺电子、高配位的 $U(V) \equiv \mathrm{N}$ 配合物 12 与 $\mathrm{H}_{2}$ 反应得到 相应的三价铀配合物 25, 该反应经历了 1,2-氢加成并发 生 1,1-氢迁移的过程(图 10). 当在氢化过程中加入缺电 子的受阻路易斯酸碱对 Frustrated Lewis Pair (FLP)的三 (2,4,6-三甲基苯基)硼时, 得到四价铀的配合物 15 和硼 复合物自由基. 12 可以直接和弱的路易斯酸 $\mathrm{BPh}_{3}$ 形成
硼的配合物 26 [U- $\mathrm{N}$ 的键长为 $0.1911(6) \mathrm{nm}$ ], 然而其 并不能发生氢化反应. 12 和位阻较大的三(2,4,6-三甲基 苯基)硼并不能形成嗍的配合物. 该工作建立了钶系元 素和 FLP 化学的独特结合并促进了氨合成的反应循环.

目前, 铀-氮多重键的研究已经取得较大进展. 但 是其他钣系元素, 比如针的多重键的配合物研究相对较 少，特别是针氮三重键配合物的合成仍然没有实现. 2019 年, Liddle 课题组 ${ }^{[16]}$ 以 $\mathrm{Th}\left(\mathrm{Tren}^{\mathrm{TIPS}}\right) \mathrm{Cl}$ 为原料, 首先 通过盐消除得到 $T h-\mathrm{N}_{3}$ 的配合物 28. 28 在甲苯溶剂中经 过 $\mathrm{KC}_{8}$ 还原，则意外得到 $\mathrm{Th}-\mathrm{CH}_{2} \mathrm{Ph}$ 的配合物 29; 以苯 为溶剂时他们认为得到了一种瞬态的 $\mathrm{Th} \equiv \mathrm{NK}_{2}$ 配合物 33. 由于 33 中的 $\mathrm{Th} \equiv \mathrm{N}$ 三键不稳定, 具有较高的反应 活性, 会进一步活化溶剂中的 $\mathrm{C}-\mathrm{H}$ 键, 产生可分离的 针-亚胺配合物 31M [Th-N 的键长: 31Li, 0.2209(2) nm; 31Na, 0.2158(5) nm; 31K, 0.2147(4) nm; 31Rb, 0.2149(6) $\mathrm{nm} ; \mathbf{3 1 C s}, 0.2105(13) \mathrm{nm}$. 配合物 31M 也可通过 32 㨦 氢的方式实现；但是如果采用甲苯为溶剂，该反应则是 可逆的. 他们以瞬态的 33 和氯硅烷反应, 通过化学转化 的方式得到了二硅胺的小分子化合物 27, 进一步证明 了 33 的存在. 28 与强路易斯酸 $\mathrm{B}\left(\mathrm{C}_{6} \mathrm{~F}_{5}\right)_{3}$ 配位可以形成配 合物 30, 但是 30 并不能通过 $\mathrm{KC}_{8}$ 还原的方式脱除 $\mathrm{N}_{2}$ 形 成多重键(图 11). 作者认为 $\mathrm{Th} \equiv \mathrm{N}$ 三键比 $\mathrm{U} \equiv \mathrm{N}$ 三键稳 定性差的原因可能与铀化学键具有更大的共价性有关. (a)

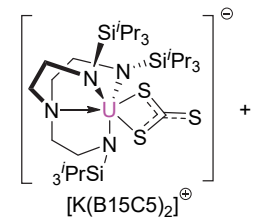

21

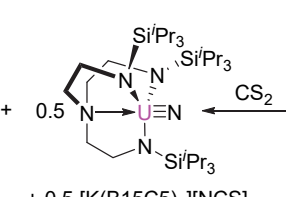

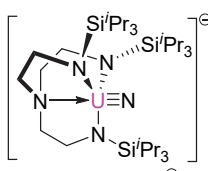

$\left[\mathrm{K}(\mathrm{B} 15 \mathrm{C} 5)_{2}\right]^{\oplus}$

12

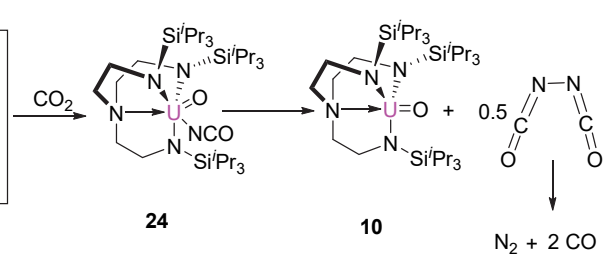

图 $9 \mathrm{U}(\mathrm{V} / \mathrm{VI}) \equiv \mathrm{N}$ 配合物与 $\mathrm{CO}_{2}$ 和 $\mathrm{CS}_{2}$ 的反应性研究

Figure 9 Study the reactivity of the terminal uranium(V/VI) nitride complexes with $\mathrm{CO}_{2}$ and $\mathrm{CS}_{2}$

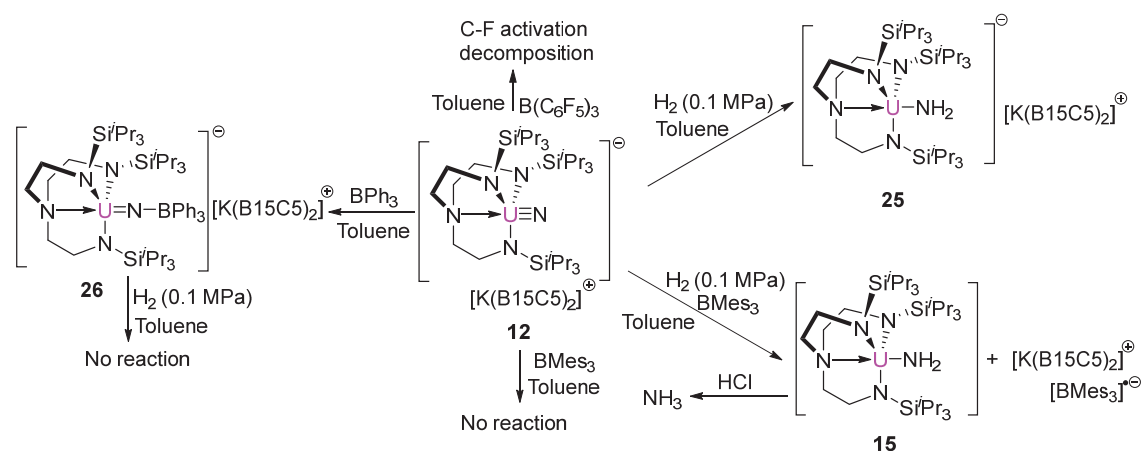

图 $10 \mathrm{U} \equiv \mathrm{N}$ 三重键配合物与禁阻路易斯酸碱对相互作用合成氨

Figure 10 Treatment of uranium nitride complexes with frustrated Lewis pair promoted ammonia synthesis 


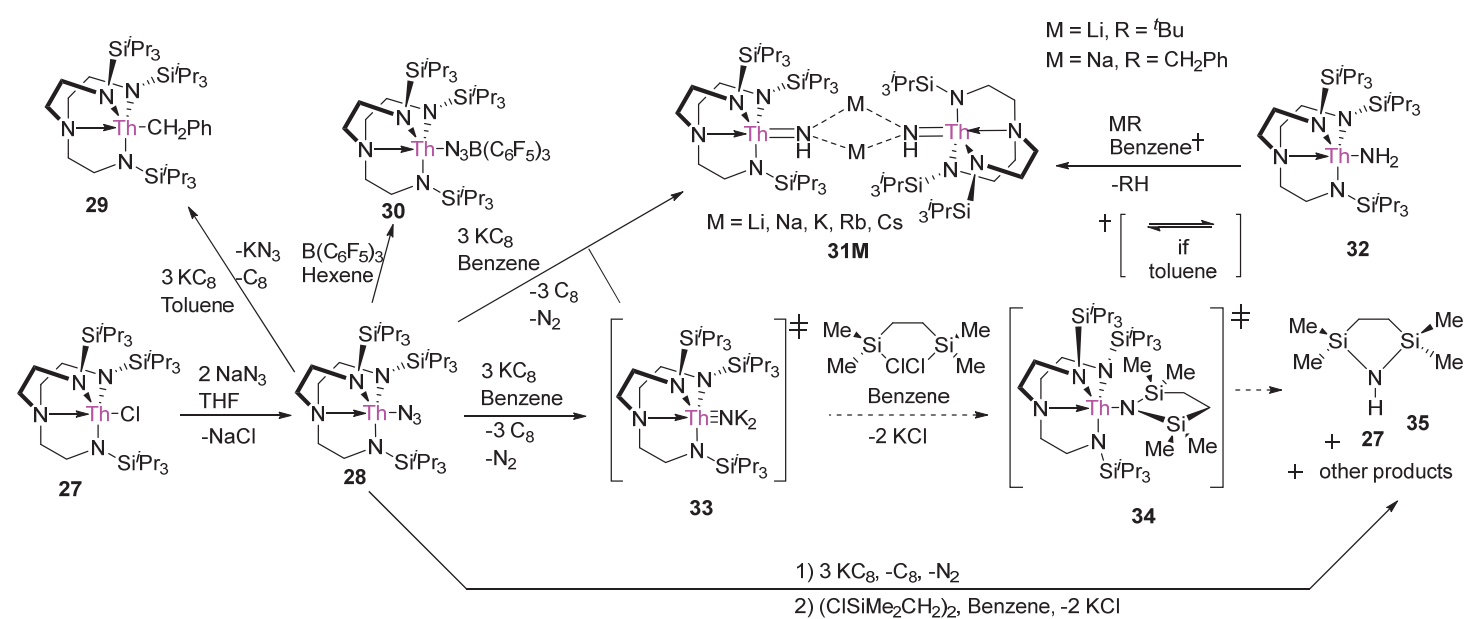

图 11 瞬态 $T h(I V) \equiv N$ 配合物的合成

Figure 11 Syntheses of transient thorium(IV) nitride complexes

除铀-氮多重键的研究外, 氮同主族的磷和砷与钣 系元素形成多重键的研究也有报道. 2014 年, 如图 12 所 示, Liddle 课题组 ${ }^{[17]}$ 采用离子性较强的 [U(Tren $\left.{ }^{\mathrm{TIPS}}\right)$ (THF)][( $\left.\left.\mathrm{BPh}_{4}\right)\right]$ 与 $\mathrm{NaPH}_{2}$ 反应, 通过脱除氯化钠, 得到配 合物 37; 而 $\left[\mathrm{U}\left(\mathrm{Tren}^{\mathrm{TIPS}}\right)(\mathrm{Cl})\right]$ 与 $\mathrm{NaPH}_{2}$ 反应来制备 37 并 没有成功, 主要原因是底物 $\mathrm{U}-\mathrm{Cl}$ 键比较强, 四价铀是 较硬的路易斯酸, 而 $\mathrm{NaPH}_{2}$ 是较软的路易斯碱, 所以

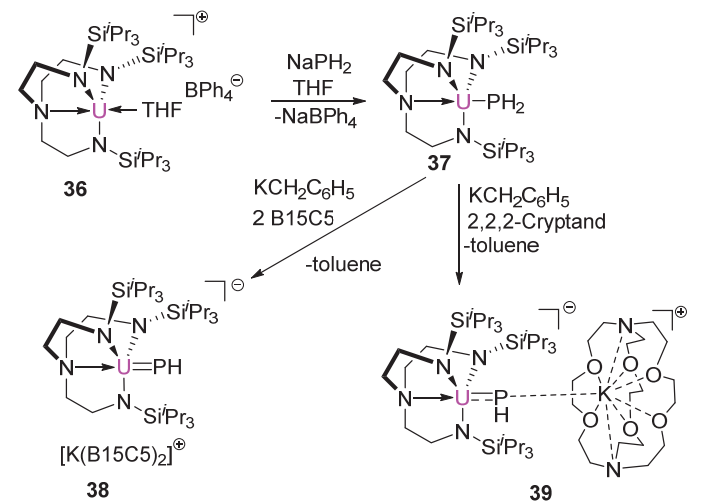

图 12 铀的端位亚磷基配合物的合成

Figure 12 Syntheses of uranium(IV) phosphinidene complexes
$-\mathrm{PH}_{2}$ 不能有效地取代 $\mathrm{Cl}$. 化合物 37 进一步通过强碱拔 氢, 并加入相应的冠醚或者穴醚, 得到电荷分离的含有 $\mathrm{U}=\mathrm{P}$ 双键的配合物 $38[\mathrm{U}-\mathrm{P}$ 的键长为 $0.2613(2) \mathrm{nm}]$ 和 39.

2017 年, Liddle 课题组 ${ }^{[18]}$ 继续深入研究了 U-P 配 合物 $\mathbf{3 7}$ 的反应性. 如图 13 所示, $\mathbf{3 7}$ 和离子对的底物 $\mathbf{3 6}$ 在强碱茮钾存在的情况下反应得到双核铀的磷配合物 40. 40 通过苠钾脱质子并加入相应的冠醚, 得到电荷分 离的包含 $\mathrm{U}=\mathrm{P}$ 双键的双核铀配合物 $43\left[\mathrm{R}=\mathrm{Si}^{i} \mathrm{Pr}_{3}, \mathrm{U}-\right.$ $\mathrm{P}$ 的键长为 $0.2653(4) \mathrm{nm}$ 和 $0.2665(4) \mathrm{nm} ; \mathrm{R}=\mathrm{SiMe}_{2}{ }^{t} \mathrm{Bu}$, $\mathrm{U}-\mathrm{P}$ 的键长为 $0.2657(2) \mathrm{nm}$ 和 $0.2713(2) \mathrm{nm}$ ]. 当然配合 物 43 也可以采用 41 和高活性 U-C 配合物的前体配合 物 42 反应得到, 反应过程伴随着 $U-C$ 键的断裂和 $U-$ $\mathrm{P}$ 键的形成. 尽管配合物 $\mathbf{4 3}$ 可以制备和分离, 但在溶液 中会迅速分解, 说明了这类配合物的不稳定性.

鉴于 $\operatorname{Tren}^{\mathrm{TIPS}}$ 配体成功用于合成 $U=N 、 U \equiv N$ 、 $\mathrm{U}=\mathrm{P}$ 化合物的经验, 为了进一步探讨铀-配体多重键的 共价性, Liddle 课题组进一步研究了铀与较软碱一砷化 合物的合成以及成键性质. 2015 年, 他们 ${ }^{[19]}$ 报道合成了 具有单键、双键和三重键铀(IV)-砷配合物, 采用离子对

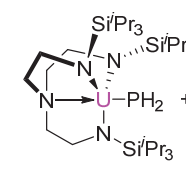

37

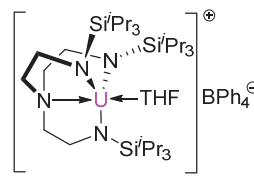

36

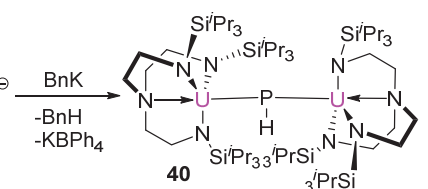

2 B15C5 $\mid-B n H$

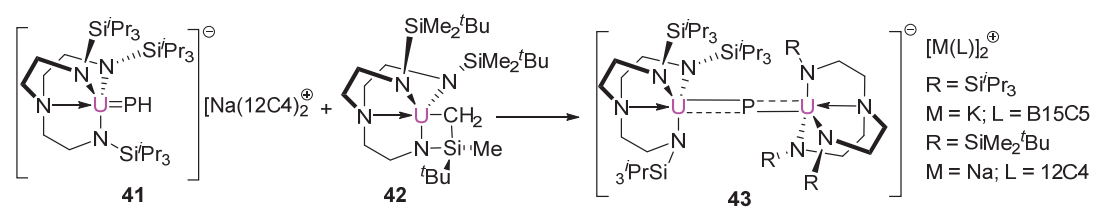

图 13 具有 $U=P$ 结构双核铀配合物的合成

Figure 13 Syntheses of dinuclear uranium complexes with UPU core 
的底物 36 和 $\mathrm{KAsH}_{2}$ 通过盐消除反应得到铀(IV)-砷单键 的配合物 44, 44 和苠钾反应 $2 \mathrm{~h}$ 脱除 As 上的质子并加 入冠醚螯合钾离子得到电荷分离的铀砷双键的配合物 45 [U-As 的键长为 $0.27159(13) \mathrm{nm}$ ], 如果反应时间延 长至两天则得到三砷的双核铀配合物 46 . 然而, 当 45 和 2 equiv. 苠钾反应时则得到罕见的铀砷三键配合物 47 [U一 As 的键长为 $0.27511(19) \mathrm{nm}$, 而且有两个钾离子 进行抗衡, 该配合物以四聚体的形式存在. 47 和冠醚反 应, 会从溶剂中篗取一个氢, 通过氢原子转移得到电荷 分离的铀砷双键的配合物 48. 但是用 $\mathrm{PbI}_{2}$ 氧化 47 并没 有得到预期的中性铀砷三键配合物, 而是发生了分子内 的 $\mathrm{C}-\mathrm{H}$ 键活化形成了新的 $\mathrm{U}-\mathrm{C}$ 键配合物 49(图 14). 理论计算表明, U-As 键比较弱, 配合物中的铀和砷之 间存在极化的共价相互作用，但通过空间位阻合适的支 撑型配体的螯合可以很好地稳定这些 U-As 多重键的 配合物. 与铀-氮三重键配合物类似, $\mathrm{U}-\mathrm{As}$ 多重键中铀 的 $5 \mathrm{f}$ 轨道而不是 $6 \mathrm{~d}$ 轨道参与成键. 该研究拓展了对钣 系配体多重键中铀与软配体成键性质的理解, 也为钶系 元素分离中的软配体设计提供了指导.
配体的微观调控有可能在反应结果上产生较大差 异. Liddle 课题组前期的工作都是采用大位阻的三异丙 基硅基保护的四价铀前体为原料开展相关工作，2019 年，他们 ${ }^{[20]}$ 采用位阻稍小的二甲基叔丁基硅基保护的 四价铀前体为原料和叠氮化钠发生盐消除得到了双核 铀的叠氮化合物 50 , 而 50 继续用 $\mathrm{KC}_{8}$ 还原可得到 $\mathrm{UNU}$ 结构的双核铀的多重键配合物 51 [ $\mathrm{U}-\mathrm{N}$ 的平均键长为 0.20648(2) nm], 这和结构相似的 8 的还原具有较大的差 异. 配合物 51 中的两个铀都为四价, 继续与 $\mathrm{AgBPh}_{4}$ 反 应，可得到四价和五价混合价态的双核铀配合物 $\mathbf{5 2}$ [U- $\mathrm{N}$ 的键长为 $0.2081(5) \mathrm{nm}$ 和 $0.2136(5) \mathrm{nm}$ ]. 而且配 合物 52 也可采用高压录灯照射 50 脱氮气的方法得到 (图 15). 以上策略也应用于 $\mathrm{Th}-\mathrm{N}_{3}$ 配合物的合成及其还 原反应研究, 但是并没有实现 $\mathrm{Th}$ 多重键配合物的构筑.

2020 年, Liddle 课题组 ${ }^{[21]}$ 采用活性的 U-C 前体化 合物 53 分别与磷叶立德和砷叶立德的底物反应得到 $\mathrm{U}=\mathrm{C}$ 多重键的配合物 54 [当 $\mathrm{E}=\mathrm{As}$ 时, $\mathrm{U}-\mathrm{C}$ 的键长为 $0.2272(6) \mathrm{nm}$; 当 $\mathrm{E}=\mathrm{P}$ 时, $\mathrm{U}-\mathrm{C}$ 的键长为 $0.2313(3) \mathrm{nm}$ ] (图 16). 在该结构中, 碳以卡宾的形式与铀配位, 而且

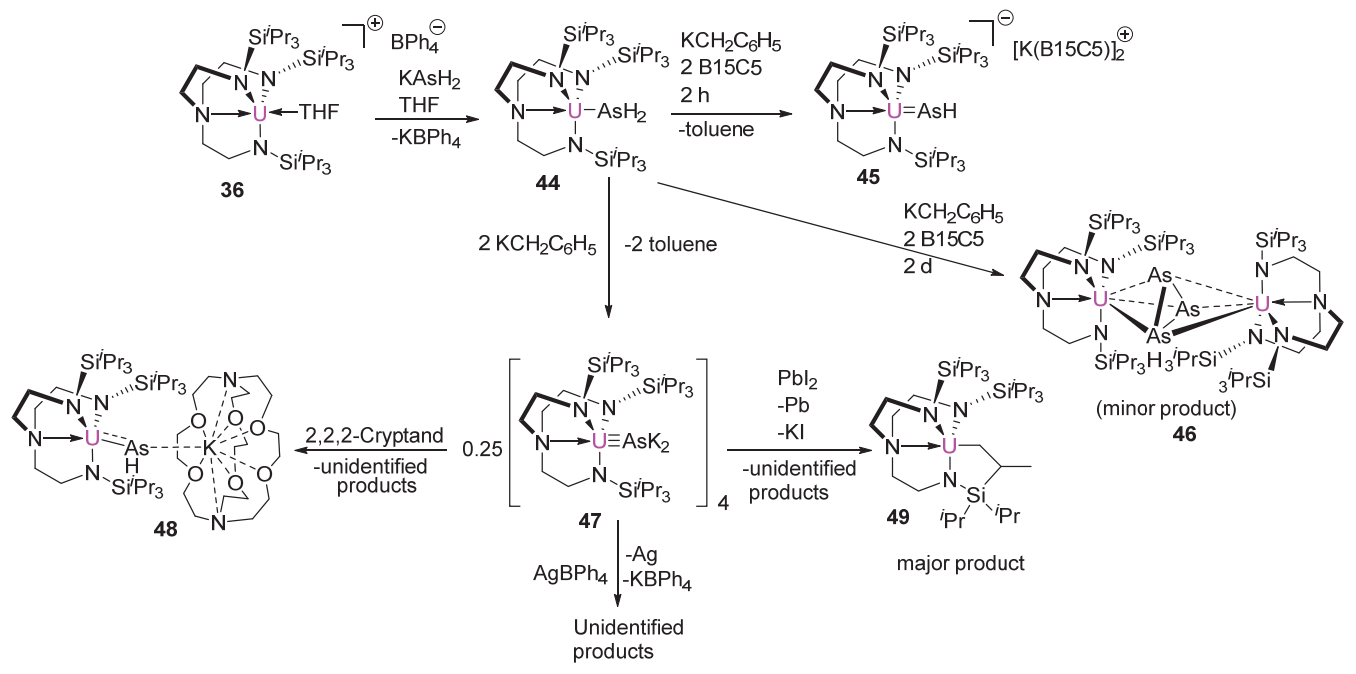

图 14 铀-砷多重键配合物的合成

Figure 14 Syntheses of uranium-arsenide multiple bond complexes

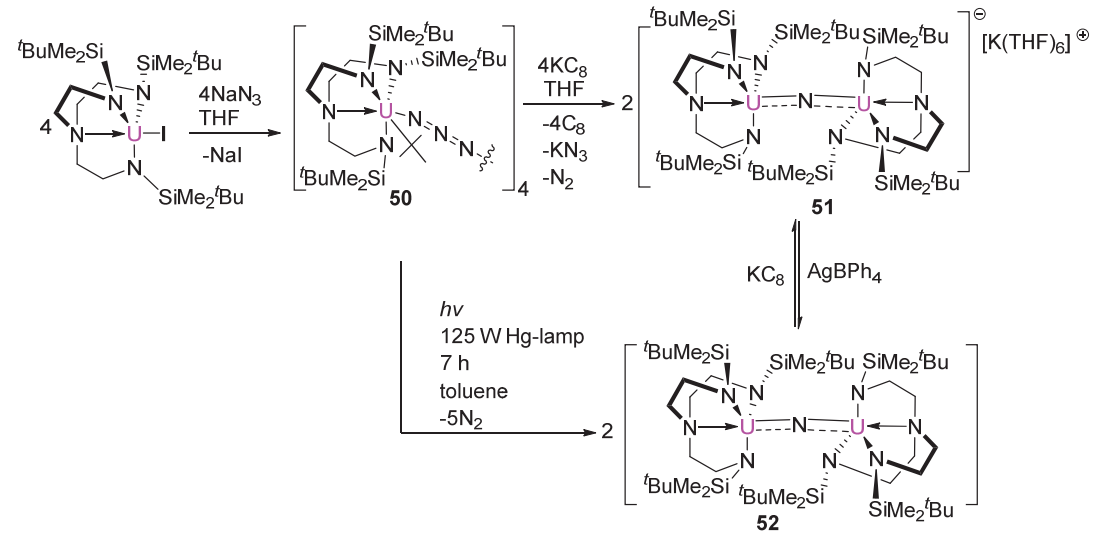

图 15 双核铀-氮多重键配合物的合成

Figure 15 Syntheses of dinuclear uranium-nitride multiple bond complexes 


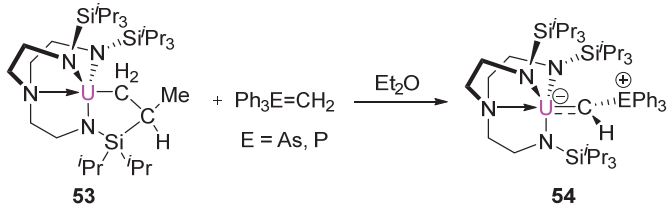

图 16 铀-砷和铀-磷卡宾配合物的合成

Figure 16 Syntheses of uranium arsonium- and phosphonium-carbene complexes

$\mathrm{U}=\mathrm{C}$ 是极化的共价键, 磷和砷并没有与铀配位. 实验 结合理论计算表明砷配合物电荷分离的偶极共振比磷 配合物强，但稳定性差.

近年来，尽管诸多铀-配体多重键的化合物已经被 报道, 但涉及其他钣系多重键配合物的研究仍然很少. 其中钍元素主要利用 $6 \mathrm{~d}$ 轨道成键, 而铀的化学键更倾 向于利用 $5 \mathrm{f}$ 轨道成键. 为了进一步研究针元素的多重 键化学性质, 2016 年, Liddle 课题组 ${ }^{[22]}$ 采用离子对的底 物 55 和 $\mathrm{NaPH}_{2}$ 通过盐消除反应制备了重要的中间产物 56(图 17). 56 进一步通过强碱拔氢并加入冠醚螯合碱金 属离子得到含有针磷双重键的配合物 57 [ Th $-\mathrm{P}$ 的键长 为 $0.27584(18) \mathrm{nm}] .57$ 和具有 $\mathrm{Th}-\mathrm{C}$ 键的高活性底物 $\mathbf{5 8}$ 反应得到具有 ThPTh 结构单元的双核钍的双重键配合 物 59 [ $\mathrm{Th}-\mathrm{P}$ 的键长为 $0.2740(2) \mathrm{nm}$ 和 $0.2735(2) \mathrm{nm}$ ]. 而 58 和不同比例的 $\mathrm{NaPH}_{2}$ 反应时根据反应的进行程度 分别得到化合物 57 和 59. 一般认为铀的成键主要是 $5 \mathrm{f}$ 轨道参与, 而钍是 $6 \mathrm{~d}$ 轨道参与成键, 但计算结果表明钍 的成键方式极其复杂微妙. 这种复杂性不仅在于 $5 \mathrm{f}$ 和 $6 \mathrm{~d}$ 轨道的参与程度, 重要的是 $7 \mathrm{~s}$ 轨道也参与了成键, 这 种成键也会影响 $5 \mathrm{f}$ 和 $6 \mathrm{~d}$ 轨道的成键特性.

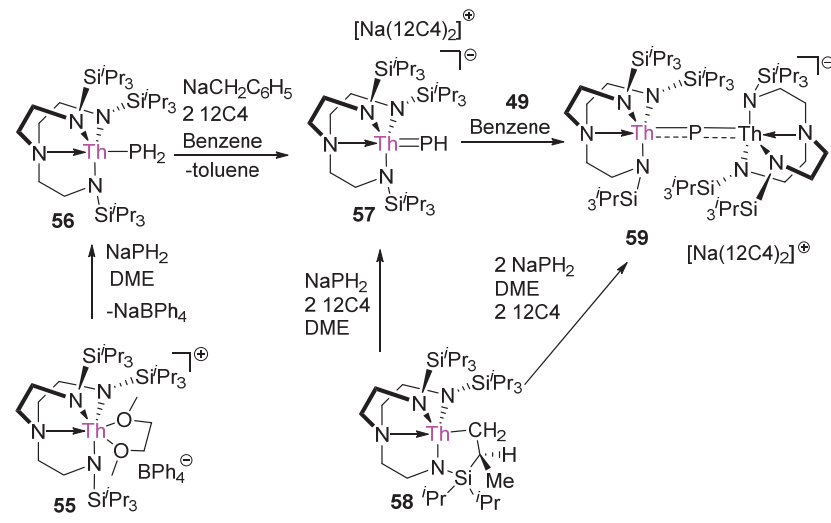

图 $17 \mathrm{Th}-\mathrm{P}$ 多重键配合物的合成

Figure 17 Syntheses of thorium-phosphorus multiple bond complexes

在前期工作的基础上, 如图 18 所示, 2017 年 Liddle 课题组 ${ }^{[23]}$ 继续对钍-砷多重键展开相关研究. 但在此之 前, 仅有两例钍-砷单键的配合物被报道 ${ }^{[24-25]}$. 这反映出 离子半径较大的钍离子在多重键的稳定极化方面仍然 有很大的挑战. 他们采用活性的 $\mathrm{Th}-\mathrm{C}$ 前体化合物 58 和 $\mathrm{KAsH}_{2}$ 反应得到了 $\mathrm{ThAsTh}$ 结构单元的双核针的双重

键配合物 60 [Th-As 的平均键长为 $0.28060(14) \mathrm{nm}]$. 计 算结果表明, $\mathrm{Th}$ 与 $\mathrm{As}$ 之间显示出极化的共价性, 这在 $\mathrm{f}$ 区元素是首例; 而且, $7 \mathrm{~s} 、 6 \mathrm{~d}$ 和 $5 \mathrm{f}$ 轨道都对 $\mathrm{Th}-\mathrm{As}$ 之 间的共价键有贡献，这与 $\mathrm{Th}-\mathrm{P}$ 的轨道贡献不同.

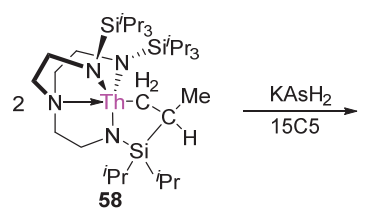

图 $18 \mathrm{Th}-\mathrm{As}$ 多重键配合物的合成

Figure 18 Synthetic routes to thorium-arsonium multiple bond complexes

\section{3 基于 Tacn 配体的钣系-配体多重键}

2006 年, Meyer 课题组 ${ }^{[26]}$ 采用大位阻酚氧配体螯合 的三价铀配合物为底物，和三甲基叠氮硅烷反应，得到 了铀氮多重键的配合物 62(图 19). 文献数据表明 $\mathrm{U} \equiv \mathrm{N}$ 三重键配合物的键长在 $0.185 \sim 0.201 \mathrm{~nm}$ 之间, 同时 $\mathrm{U}-\mathrm{N}-\mathrm{X}$ 的键角处于 $163.33^{\circ} \sim 180.0^{\circ}$. 单晶结构表明, 当 $\mathrm{R}$ 为叔丁基时, $\mathrm{U}-\mathrm{N}$ 键长为 $0.1989(5) \mathrm{nm}, \mathrm{U}-\mathrm{N}-\mathrm{Si}$ 的键 角为 $173.7(3)^{\circ}$, 这和 $\mathrm{U} \equiv \mathrm{N}$ 三重键配合物的数据非常接 近 [27]; 当 $\mathrm{R}$ 为金刚烷取代基时, $\mathrm{U}-\mathrm{N}$ 键长为 $0.21219(18) \mathrm{nm}, \mathrm{U}-\mathrm{N}-\mathrm{Si}$ 的键角为 $162.55(12)^{\circ}$. 配合物 62 由于拥有空间位阻较大的金刚烷基团，致使形成较 狭窄的空腔, 阻碍了铀与 NTMS 的有效键合. 因此, 氮 的 $\mathrm{p}$ 轨道不能有效地参与金属-配体的 $\pi$ 键，从而导致配 合物 62 一些不同寻常的结构参数.
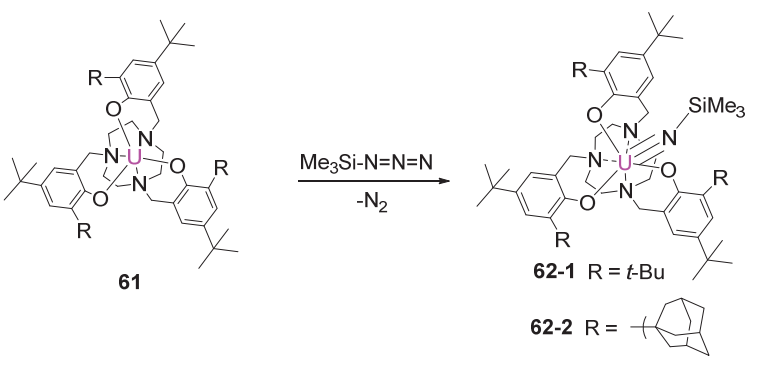

图 19 三价铀促进的铀氮三重键配合物的合成

Figure 19 Trivalent uranium precursor complex promoted syntheses of uranium nitride complexes

2008 年, Meyer 课题组 ${ }^{[28]}$ 研究发现三价铀配合物 61 可以有效还原芳基叠氮得到高价铀(V)亚氨基配合物 63 $\left[\mathrm{R}={ }^{t} \mathrm{Bu}, \mathrm{U}-\mathrm{N}\right.$ 的键长为 $\left.0.2047(8) \mathrm{nm}\right]$ (图 20). 化合物 63 具有相对较长的 $U-N$ 键, 具有很强的反应活性, 进 而发生二氧化碳插入反应得到配合物 $64\left[\mathrm{R}={ }^{t} \mathrm{Bu}, \mathrm{U}-\mathrm{O}\right.$ 的键长为 $0.1848(8) \mathrm{nm} ; \mathrm{R}=\mathrm{Ad}, \mathrm{U}-\mathrm{O}$ 的键长为 $0.1848(4) \mathrm{nm}]$. 晶体结构证实 64 为末端 $U \equiv \mathrm{O}$ 三重键化 合物. 随后, 作者 ${ }^{[29]}$ 对 64 的反应性进行了研究, $\mathrm{AgSbF}_{6}$ 可进一步将其氧化为六氟合锑酸根的 $U(V I) \equiv \mathrm{O}$ 配合物 65 和 $66[\mathrm{U}-\mathrm{O}$ 的键长为 $0.1836(6) \mathrm{nm}], \mathrm{AgO}_{2} \mathrm{CCF}_{3}$ 作为 
氧化剂时, $\mathrm{CF}_{3} \mathrm{CO}_{2}{ }^{-}$可以键合到 $\mathrm{U}(\mathrm{VI})$ 上形成相关配合物 67 [U-O 的键长为 $0.1811(2) \mathrm{nm}$ ].

2014 年, Meyer 课题组 ${ }^{[30]}$ 发现氧气可直接氧化三价 铀的配合物 68 生成具有金刚烷结构的双核 $U(V) \equiv O$ 配 合物 69 [U一O 的键长为 $0.20354(12) \mathrm{nm}$ 和 $0.21815(11)$ $\mathrm{nm}$, 也可被吡啶氮氧化物和三级胺的氮氧化物直接氧 化三价铀的配合物为 $\mathrm{U}(\mathrm{V}) \equiv \mathrm{O}$ 配合物 70 [U一 $-\mathrm{O}$ 的键长 为 $0.1856(2) \mathrm{nm}$ ]和 71 [U-O 的键长为 $0.1860(2) \mathrm{nm}] .68$ 和芳基叠氮反应, 通过脱除氮气, 得到 U(V)亚胺配合物 72 [U- $\mathrm{N}$ 的键长为 $0.1979(3) \mathrm{nm}], 72$ 继续和二氧化碳反 应也可以转化为 69 . 配合物 69 通过 $\mathrm{KC}_{8}$ 还原, 分别得 到电荷分离的配合物 $73[\mathrm{U}-\mathrm{O}$ 的平均键长为 $0.21445(3) \mathrm{nm}$ ]和金属钾配位的配合物 75 [U一 $-\mathrm{O}$ 的平均 键长为 $0.21661(16) \mathrm{nm}] ; 69$ 通过三氟磺酸银氧化得到 74, 而 73 也可通过三氟磺酸银氧化得到 74 [U-O 的键 长为 $0.1794(2) \mathrm{nm}], 75$ 通过 $\mathrm{AgSbF}_{6}$ 氧化得到 $\mathrm{U}(\mathrm{VI}) \equiv \mathrm{O}$ 的配合物 76 [U一O 的键长为 $0.1791(6) \mathrm{nm}]$, 而 76 通过 $\mathrm{KC}_{8}$ 还原重新得到 75 实现该反应的可逆性(图 21).

2014 年, Meyer 课题组 ${ }^{[31]}$ 报道了一系列不同氧化态 $(\mathrm{U}(\mathrm{IV}) 、 \mathrm{U}(\mathrm{V})$ 和 $\mathrm{U}(\mathrm{VI}))$ 的多重键配合物. 该系列是由三 价铀前体 77 通过多步转化而来. 77 和有机叠氮化合物 trityl- $\mathrm{N}_{3}$ 反应得到 $\mathrm{U}(\mathrm{V}) \equiv \mathrm{N}$ 三重键的配合物 $78[\mathrm{U}-\mathrm{N}$ 的键长为 $0.1939(3) \mathrm{nm}], 78$ 和 $\mathrm{CO}_{2}$ 反应得到双核的 $U(V)$ $=\mathrm{O}$ 配合物 81.81 也可由 77 和 $80\left(\mathrm{Mes}-\mathrm{N}_{3}\right) 、 \mathrm{CO}_{2}$ 一锅
法来构筑. 78 被银离子氧化得到 $\mathrm{U}(\mathrm{VI}) \equiv \mathrm{N}$ 三重键的配 合物 79 [U-N 的键长为 $0.1920(2) \mathrm{nm}]$. 而 78 被 $\mathrm{KC}_{8}$ 还 原得到钾离子螯合的 $\mathrm{U}(\mathrm{IV})=\mathrm{N}$ 配合物 $82[\mathrm{U}-\mathrm{N}$ 的键长 为 0.2036(2) nm], 82 可被 $\mathrm{CAN}$ 质子化得到配合物 83.78 在热的甲苯溶液中搅拌 $2 \mathrm{~d}$ 也可以得到配合物 83 . 而 83 进一步和 $\mathrm{CO}_{2}$ 反应得到铀氧多重键配合物, 并同时生成 异氧酸酯(图 22). 研究结果表明, U(VI)氮化物不与 $\mathrm{CO}_{2}$ 反应，而 $U(V)$ 和 $U(I V)$ 氮化物发生复分解反应.

2016 年, Meyer 课题组 ${ }^{[32]}$ 报道了一系列 $\mathrm{U} \equiv \mathrm{S}$ 三重 键配合物的合成. $\mathrm{H}_{2} \mathrm{~S}$ 与 U(III)的配合物 85 通过脱除氢 气的还原反应得到四价铀的硫化物 86. 86 在强碱条件下 发生脱氢质子反应，同时在 18-crown-6 和 2.2.2-cryptand 的参与下, 得到相应的配合物 87 [U $-\mathrm{S}$ 的键长为 $0.2536(2) \mathrm{nm}$ ]和 88 [U-S 的键长为 $0.2507(1) \mathrm{nm}$ ](图 $23)$. 光谱和计算结果表明配合物具有 $C_{3}$ 轴对称性, 以 及配合物中化学键为 $\mathrm{U} \equiv \mathrm{S}$ 三重键的特征.

2019 年, Meyer 课题组 ${ }^{[33]}$ 在前期工作的基础上, 采 用类似的策略，合成了完整系列的末端 $U \equiv E$ 配合物 91 $[\mathrm{U}-\mathrm{O}$ 的键长为 $0.1868(5) \mathrm{nm} ; \mathrm{U}-\mathrm{S}$ 的键长为 $0.2536(2)$ $\mathrm{nm} ; \mathrm{U}-\mathrm{Se}$ 的键长为 $0.2659(2) \mathrm{nm} ; \mathrm{U}-\mathrm{Te}$ 的键长为 $0.2942(2) \mathrm{nm}]$ (图 24). 单晶 X-射线衍射、UV/vis/NIR、 电子吸收和红外振动光谱、SQUID 变温磁矩和计算分析 等多种手段探究了不同末端 $U \equiv E$ 化合物 $(E=O 、 S 、 S e 、$ $\mathrm{Te})$ 的电子结构所产生的影响以及其周期性的规律.

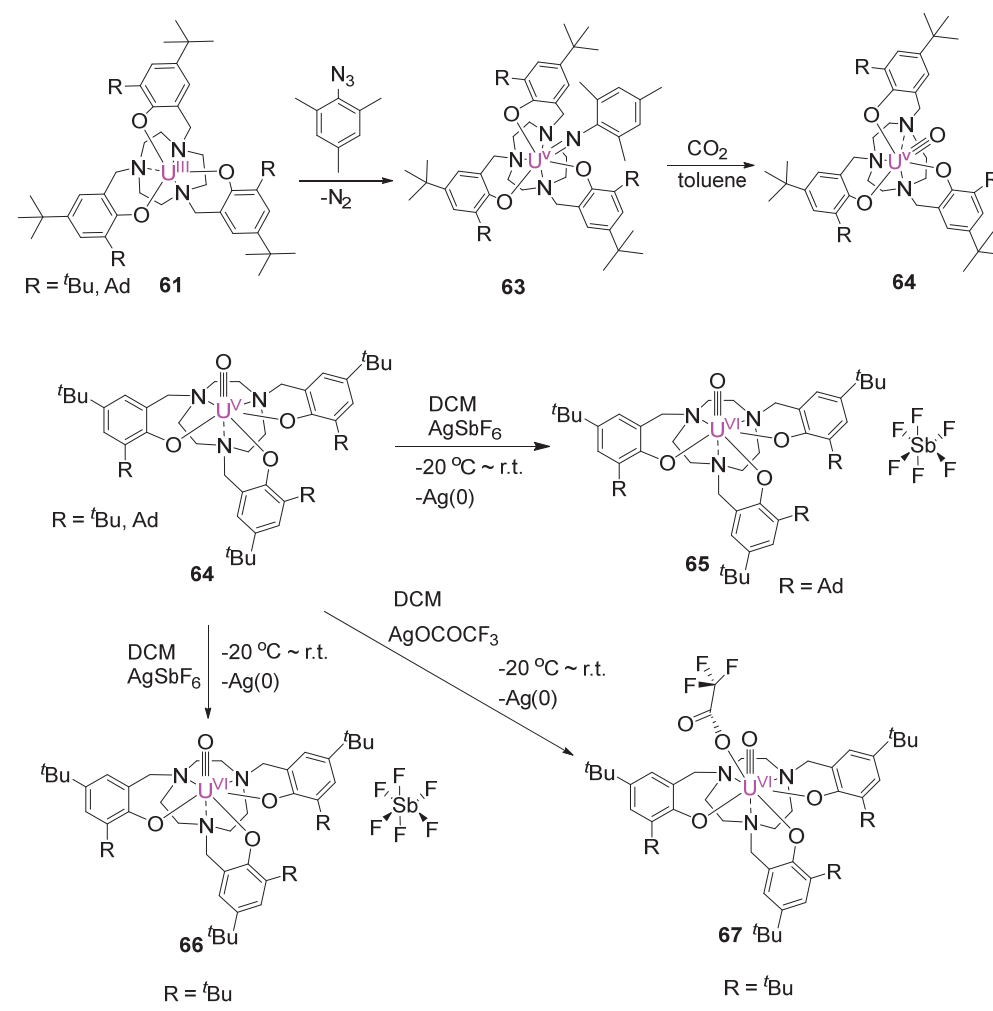

图 $20 \mathrm{U}(\mathrm{V})=\mathrm{N}$ 和 $\mathrm{U}(\mathrm{V}) \equiv \mathrm{O}$ 配合物的合成及其反应性研究

Figure 20 Syntheses and reactivity of uranium(V)-imido complexes and uranium(V)-oxo complexes 


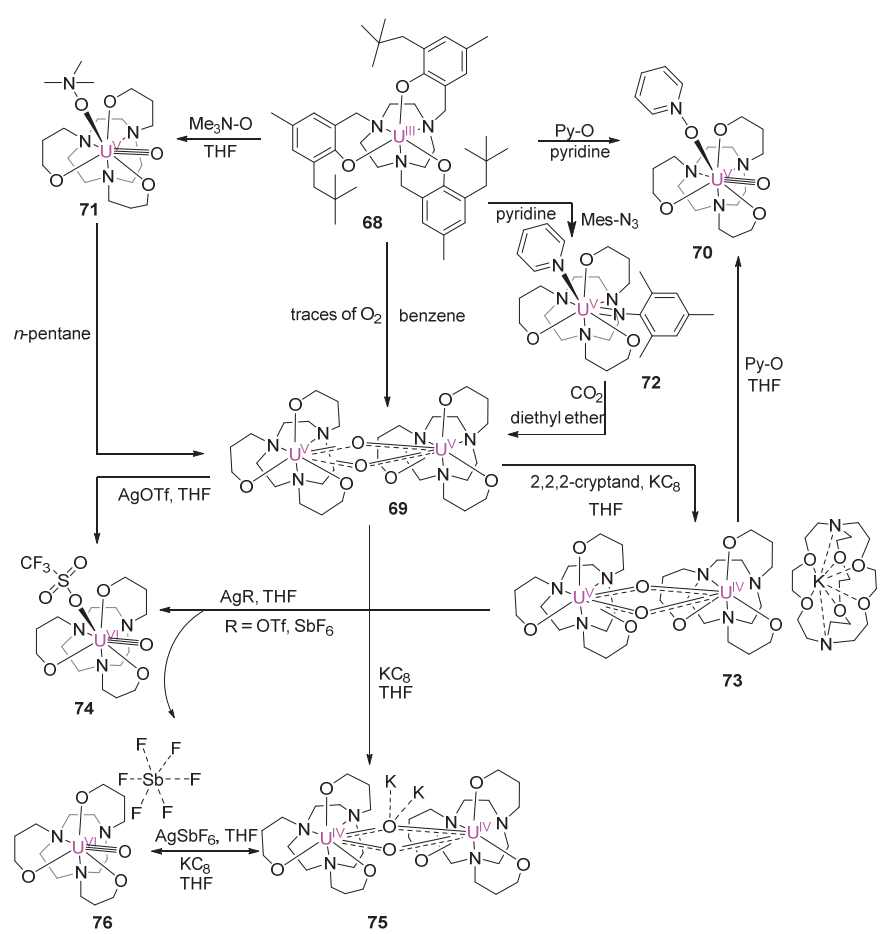

图 21 六价、五价、四价铀氧多重键的合成(戊基和甲基取代的酚氧芳环在该处进行了省略以保证结构图更清晰明了)

Figure 21 Reaction scheme for the syntheses of U(VI), U(V) and U(IV) oxo multiple bond complexes (a phenolate rings, including the neopentyl and methyl substituent, are omitted for clarity)

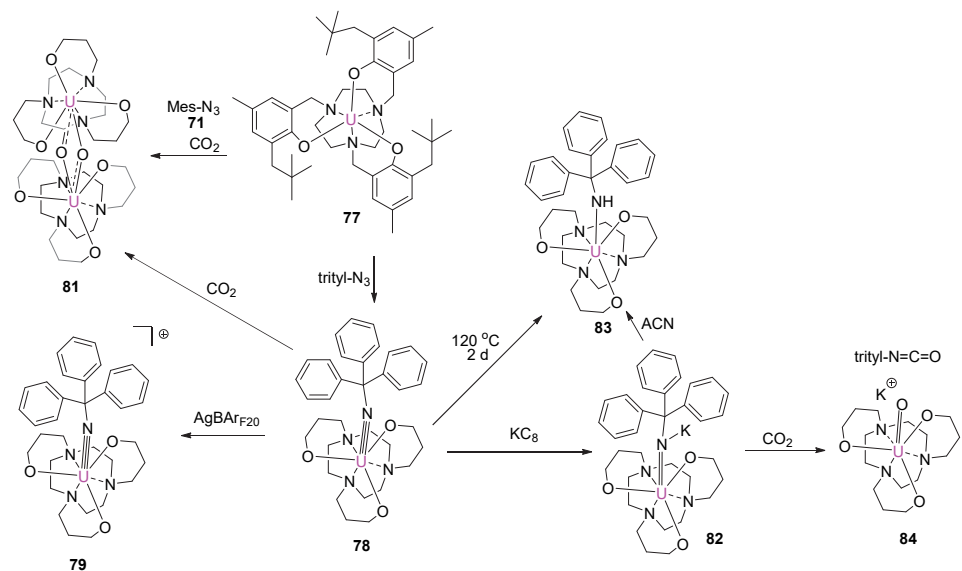

图 $22 \mathrm{U} \equiv \mathrm{N}, \mathrm{U}=\mathrm{N}$ 和 $\mathrm{U}=\mathrm{O}$ 多重键配合物的合成(戊基和甲基取代的酚氧芳环在该处进行了省略以保证结构图更清晰明了)

Figure 22 Reaction scheme for the syntheses of the uranium imido, amido and oxo multiple bond complexes (a phenolate rings, including the neopentyl and methyl substituent, are omitted for clarity)

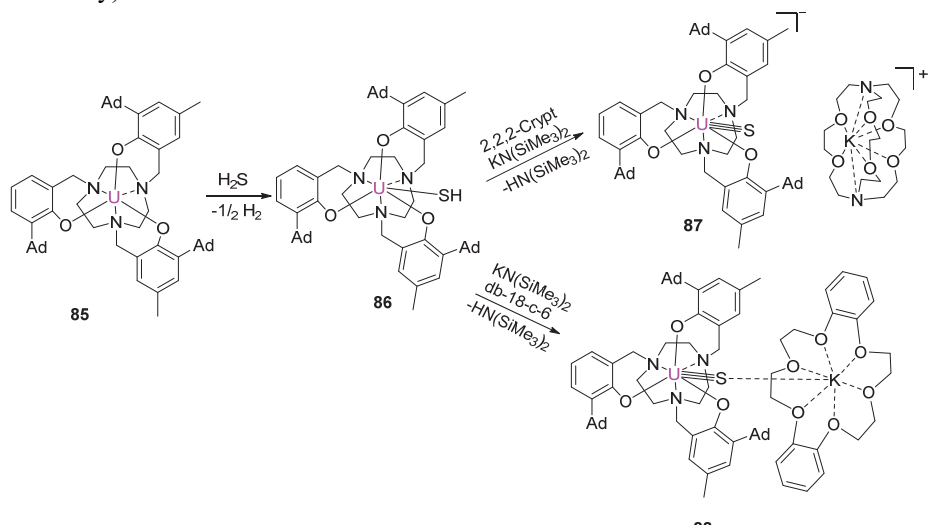

图 $23 \mathrm{U} \equiv \mathrm{S}$ 三重键配合物的合成

Figure 23 Syntheses of the terminal U(IV) $\equiv \mathrm{S}$ complexes 


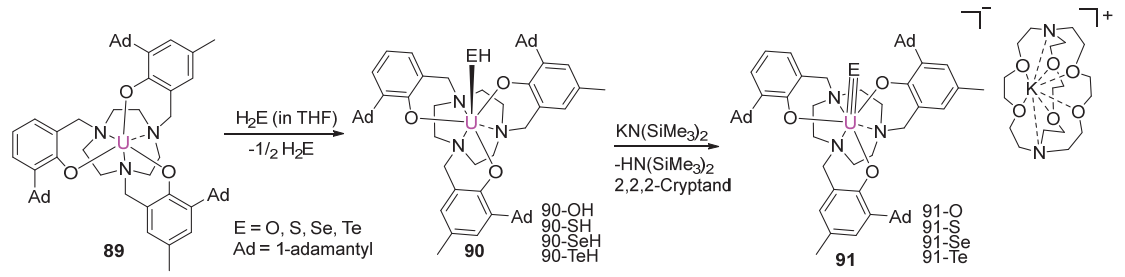

图 24 末端硫族铀三重键配合物的合成

Figure 24 Synthetic route to the terminal uranium(IV) chalcogenido triple bond complexes

三价铀具有较强的还原性, 一氧化二氮可以将其氧 化为五价铀的 $U=O$ 配合物 $93[U-O$ 的键长为 $0.1831(2) \mathrm{nm}]^{[34]}$. 配合物 93 进一步被 $\mathrm{KC}_{8}$ 还原, 得到离 子化的四价铀配合物 94 [U-O 的键长为 0.1891(6) nm]. 在 93 和 94 中, 芳烃与铀形成的 $\delta$ 键对配合物的稳定性 起着重要的作用(图 25). 上述两类配合物的合成为三价 铀电催化分解水的可能反应机理提供了重要的证据 ${ }^{[35]}$.

\section{4 基于 TriNOx 配体的钣系-配体多重键}

2015 年, Schelter 课题组 ${ }^{[36]}$ 首次成功合成了具有三 脚架结构的三氮氧配体并成功实现 $\mathrm{Nd}$ 和 $\mathrm{Dy}$ 以及 $\mathrm{Yb}$ 和 $\mathrm{Lu}$ 的分离 ${ }^{[37]}$. 由于其具有三阴离子四齿螯合的骨架结 构, 可以很好地稳定四价 $\mathrm{Ce}$, 实现了 $\mathrm{Ce}=\mathrm{N}$ 配合物的
合成 ${ }^{[38]}$. 虽然 Ce(IV)和 Th(IV)结构上具有一定的类似 性，但是其成键性质具有较大差异; 二者成键性质的差 异, 特别是共价性差异可为镧钣分离提供重要的科学依 据.

2019 年, Schelter 课题组 ${ }^{[39]}$ 通过一系列的转化实现 了 $\mathrm{Th}(\mathrm{IV})=\mathrm{N}$ 配合物 98 [ $\mathrm{Th}-\mathrm{N}$ 的键长为 $0.2205(6) \mathrm{nm}$ ] 和 99 [ Th $-\mathrm{N}$ 的键长为 $0.2149(5) \mathrm{nm}$ ]的合成, 采用实验 和理论相结合的方法研究揭示 $\mathrm{Th}(\mathrm{IV})=\mathrm{N}$ 与 $\mathrm{Ce}(\mathrm{IV})=\mathrm{N}$ 配合物的显著差异, $\mathrm{Ce}(\mathrm{IV})=\mathrm{N}$ 多重键的共价性大于 $T h(I V)=N$ 多重键(图 26). 一般认为钣系元素比镧系元 素具有更强的共价性, 而该工作中 $\mathrm{Ce}(\mathrm{IV})=\mathrm{N}$ 配合物展 现出的反常共价性由其电子结构和反应性所决定.
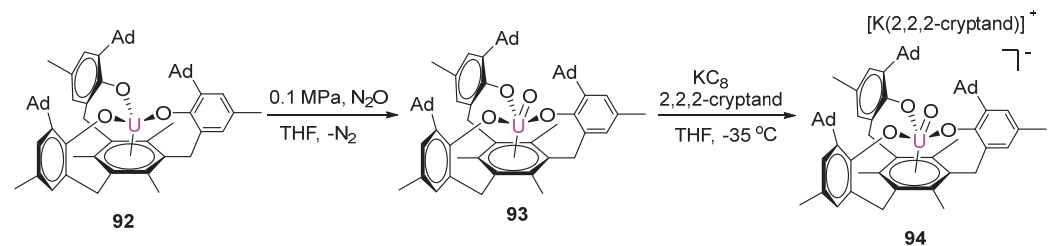

图 $25 \mathrm{U}=\mathrm{O}$ 配合物的合成

Figure 25 Syntheses of uranium oxo double bond complexes

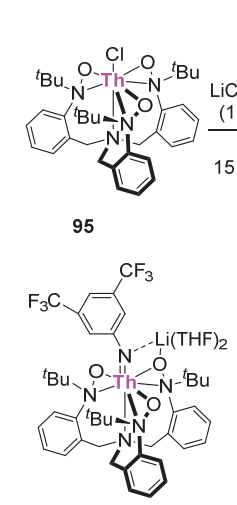

98

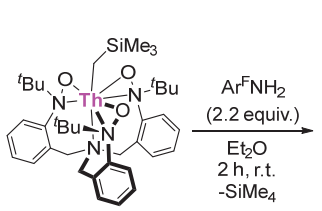

96

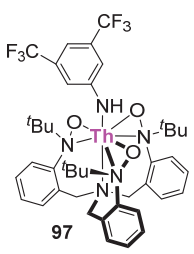

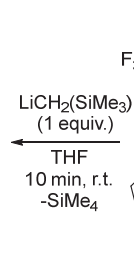

$-\mathrm{SiMe}_{4}$

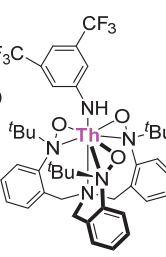

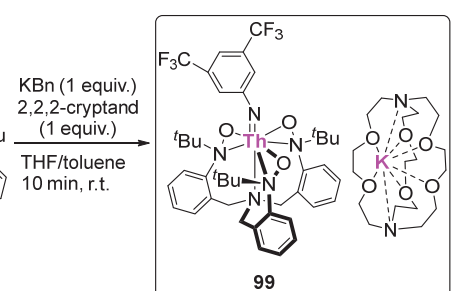

97

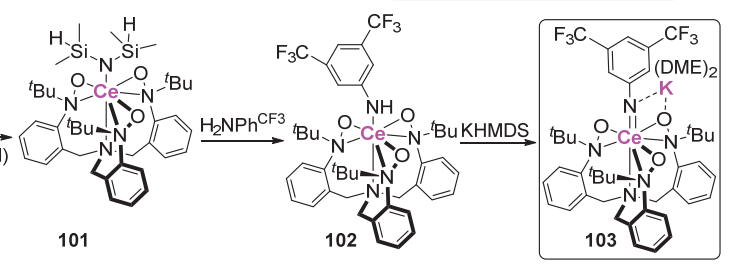

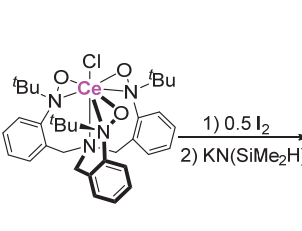

100

94

图 $26 \mathrm{Th}(\mathrm{IV})=\mathrm{N}$ 配合物和 $\mathrm{Ce}(\mathrm{IV})=\mathrm{N}$ 配合物的共价性比较

Figure 26 Comparison of covalency in thorium(IV)- and cerium(IV)-imido complexes 


\section{5 总结与展望}

综上所述, 近年来在钣系配体的合成和多重键的研 究方面取得了相当大的进展. 配体在多重键的构筑方面 有着重要的作用. 然而, 没有一个普适性的配体可以适 用于所有反应; 因此, 新型配体的设计一直是多重键配 合物合成中的重点. 而新型配体的设计合成需要考虑是 否容易制备、兼顾稳定性和反应活性以及位阻的可调节 性. 同时, 我们课题组 ${ }^{[40-43]}$ 在新型三脚架配体的设计合 成和钶系-配体多重键的理论计算方面也取得了一定的 进展. 然而, 钣系-配体多重键的研究仍然处于起始阶 段, 仍有待进一步深入探究, 比如: 如何提升空间大位 阻化合物的合成产率及如何探索温和的反应条件; 如何 扩展钣系金属配体化合物的类型; 如何探索新钶系化合 物的应用领域; 在除铀外的其他钣系元素多重键的研究 方面依然欠缺. 因此, 深入研究钣系元素化合物以及金 属配体多重键的化学性质依然是未来研究的主要内容. 研究含有钶系配体多重键的钶系有机化合物的合成、反 应和成键本质具有非常重要的科学意义和应用前景.

\section{作者简介}

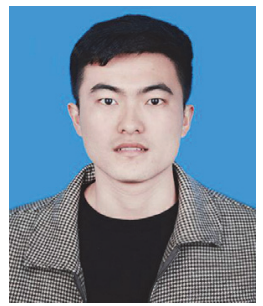

李斌, 2019 年 6 月毕业于郑州大学化学学院, 获理学学士 学位; 2019 年 9 月至今为哈尔滨工程大学和中国科学院高能物 理研究所联合培养研究生.

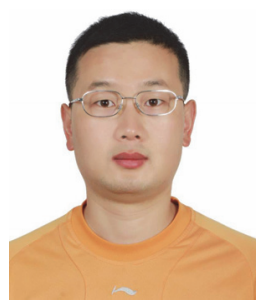

于吉攀, 博士, 助理研究员. 2008 年获江苏师范大学学士 学位; 2011 年获南开大学硕士学位; 2014 年获清华大学博士学 位; 2014.07 2016.07 清华大学化学系博士后; 2016 年 7 月加 入中国科学院高能物理研究所核能放射化学实验室. 目前主 要研究方向: 铜系元素化学.

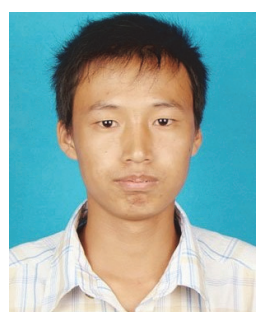

刘康, 2016 年 6 月毕业于湖北大学化学化工学院, 获理学 学士学位; 2016 年 9 月至今在中国科学院高能物理研究所攻读
博士学位.

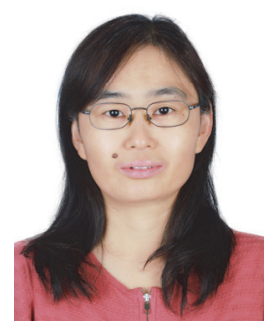

吴群燕, 博士, 副研究员. 2006 年获曲阜师范大学硕士学 位; 2010 年获北京理工大学博士学位; 2010 2013 清华大学化 学系博士后; 2013 年 6 月加入中国科学院高能物理研究所核能 放射化学实验室, 主要从事与核能相关的铜系化学理论方面 的研究工作.

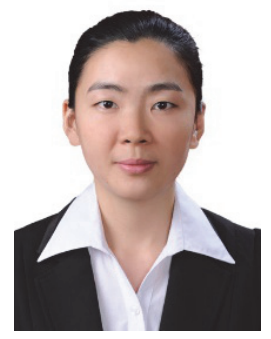

刘琦, 博士，副教授. 2007 年获哈尔滨工程大学环境工程 专业学士学位; 2009 年获哈尔滨工程大学应用化学专业硕士 学位; 2016 年获哈尔滨工程大学材料学专业博士学位; 2009 年 6 月进入哈尔滨工程大学材料科学与化学工程学院工作, 目前 主要从事海水提铀吸附剂材料的设计研究.

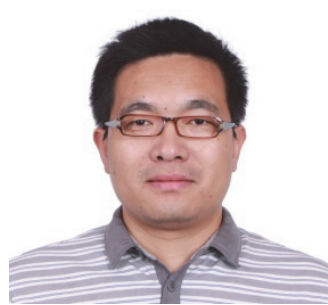

石伟群, 研究员, 国家杰出青年科学基金获得者, 长期致 力于核燃料循环化学与铜系元素化学相关基础研究, 在 JACS、Angew. Chem、Chem、CCS Chem. Nat. Commun、 Adv. Mater. Environ. Sci. Technol. 等国际知名期刊发表 SCI 论 文 200 余篇, 成果被国内外同行广泛关注和引用, 文章总引 7400 余次, H 因子 44 (Google Scholar). 分别担任英文期刊 《Journal of Nuclear Fuel Cycle and Waste Technology》和 《Journal of Nuclear Science and Technology》的编委与国际顾 问编委, 中文期刊《核化学与放射化学》编委. 现为中国化学 会核化学与放射化学专业委员会委员、中国核学会铜系物理 与化学分会常务理事、中国有色金属学会熔盐化学与技术专 业委员会副主任委员、中国核学会核化工分会理事兼副秘书 长.

\section{References}

[1] Fox, A. R.; Bart, S. C.; Meyer, K.; Cummins, C. C. Nature 2008, 455,341 .

[2] Gardner, B. M.; Liddle, S. T. Chem. Commun. 2015, 51, 10589.

[3] Lu, E.; Boronski, J. T.; Gregson, M.; Wooles, A. J.; Liddle, S. T. Angew. Chem. Int. Ed. 2018, 57, 5506.

[4] Gregson, M.; Lu, E.; Mills, D. P.; Tuna, F.; McInnes, E. J. L.; Hen- 
nig, C.; Scheinost, A. C.; McMaster, J.; Lewis, W.; Blake, A. J.; Kerridge, A.; Liddle, S. T. Nat. Commun. 2017, 8, 14137.

[5] Lu, E.; Tuna, F.; Lewis, W.; Kaltsoyannis, N.; Liddle, S. T. Chem.-Eur. J. 2016, 22, 11554.

[6] King, D. M.; Tuna, F.; McInnes, E. J. L.; McMaster, J.; Lewis, W.; Blake, A. J.; Liddle, S. T. Science 2012, 337, 717.

[7] Gardner, B. M.; Cleaves, P. A.; Kefalidis, C. E.; Fang, J.; Maron, L.; Lewis, W.; Blake, A. J.; Liddle, S. T. Chem. Sci. 2014, 5, 2489.

[8] King, D. M.; Tuna, F.; McInnes, E. J. L.; McMaster, J.; Lewis, W.; Blake, A. J.; Liddle, S. T. Nat. Chem. 2013, 5, 482.

[9] Thomson, R. K.; Cantat, T.; Scott, B. L.; Morris, D. E.; Batista, E. R.; Kiplinger, J. L. Nat. Chem. 2010, 2, 723.

[10] King, D. M.; Tuna, F.; McMaster, J.; Lewis, W.; Blake, A. J.; McInnes, E. J. L.; Liddle, S. T. Angew. Chem. Int. Ed. 2013, 52, 4921.

[11] Cleaves, P. A.; King, D. M.; Kefalidis, C. E.; Maron, L.; Tuna, F.; McInnes, E. J. L.; McMaster, J.; Lewis, W.; Blake, A. J.; Liddle, S. T. Angew. Chem. Int. Ed. 2014, 53, 10412.

[12] King, D. M.; McMaster, J.; Tuna, F.; McInnes, E. J. L.; Lewis, W.; Blake, A. J.; Liddle, S. T. J. Am. Chem. Soc. 2014, 136, 5619.

[13] King, D. M.; Cleaves, P. A.; Wooles, A. J.; Gardner, B. M.; Chilton, N. F.; Tuna, F.; Lewis, W.; McInnes, E. J. L.; Liddle, S. T. Nat. Commun. 2016, 7, 13773.

[14] Cleaves, P. A.; Kefalidis, C. E.; Gardner, B. M.; Tuna, F.; McInnes, E. J. L.; Lewis, W.; Maron, L.; Liddle, S. T. Chem.-Eur. J. 2017, 23, 2950.

[15] Chatelain, L.; Louyriac, E.; Douair, I.; Lu, E.; Tuna, F.; Wooles, A. J.; Gardner, B. M.; Maron, L.; Liddle, S. T. Nat. Commun. 2020, 11, 337.

[16] Du, J.; Alvarez-Lamsfus, C.; Wildman, E. P.; Wooles, A. J.; Maron, L.; Liddle, S. T. Nat. Commun. 2019, 10, 4203.

[17] Gardner, B. M.; Balázs, G.; Scheer, M.; Tuna, F.; McInnes, E. J. L.; McMaster, J.; Lewis, W.; Blake, A. J.; Liddle, S. T. Angew. Chem. Int. Ed. 2014, 53, 4484 .

[18] Rookes, T. M.; Gardner, B. M.; Balázs, G.; Gregson, M.; Tuna, F.; Wooles, A. J.; Scheer, M.; Liddle, S. T. Angew. Chem. Int. Ed. 2017, 56,10495 .

[19] Gardner, B. M.; Balázs, G.; Scheer, M.; Tuna, F.; McInnes, E. J. L.; McMaster, J.; Lewis, W.; Blake, A. J.; Liddle, S. T. Nat. Chem. 2015, 7, 582 .

[20] Du, J.; King, D. M.; Chatelain, L.; Lu, E.; Tuna, F.; McInnes, E. J. L.; Wooles, A. J.; Maron, L.; Liddle, S. T. Chem. Sci. 2019, 10, 3738.

[21] Seed, J. A.; Sharpe, H. R.; Futcher, H. J.; Wooles, A. J.; Liddle, S. T. Angew. Chem. Int. Ed. 2020, 59, 15870.

[22] Wildman, E. P.; Balázs, G.; Wooles, A. J.; Scheer, M.; Liddle, S. T. Nat. Commun. 2016, 7, 12884.
[23] Wildman, E. P.; Balázs, G.; Wooles, A. J.; Scheer, M.; Liddle, S. T. Nat. Commun. 2017, 8, 14769.

[24] Behrle, A. C.; Walensky, J. R. Dalton Trans. 2016, 45, 10042.

[25] Scherer, O. J.; Schulze, J.; Wolmershäuser, G. J. Organomet. Chem. 1994, 484, c5.

[26] Castro-Rodríguez, I.; Nakai, H.; Meyer, K. Angew. Chem. Int. Ed. 2006, 45, 2389.

[27] Castro-Rodriguez, I.; Olsen, K.; Gantzel, P.; Meyer, K. J. Am. Chem. Soc. 2003, 125, 4565 .

[28] Bart, S. C.; Anthon, C.; Heinemann, F. W.; Bill, E.; Edelstein, N. M.; Meyer, K. J. Am. Chem. Soc. 2008, 130, 12536.

[29] Kosog, B.; La Pierre, H. S.; Heinemann, F. W.; Liddle, S. T.; Meyer, K. J. Am. Chem. Soc. 2012, 134, 5284.

[30] Schmidt, A.-C.; Heinemann, F. W.; Lukens, W. W.; Meyer, K. J. Am. Chem. Soc. 2014, 136, 11980.

[31] Schmidt, A.-C.; Heinemann, F. W.; Maron, L.; Meyer, K. Inorg. Chem. 2014, 53, 13142.

[32] Rosenzweig, M. W.; Scheurer, A.; Lamsfus, C. A.; Heinemann, F. W.; Maron, L.; Andrez, J.; Mazzanti, M.; Meyer, K. Chem. Sci. 2016, 7, 5857.

[33] Rosenzweig, M. W.; Hümmer, J.; Scheurer, A.; Lamsfus, C. A.; Heinemann, F. W.; Maron, L.; Mazzanti, M.; Meyer, K. Dalton Trans. 2019, 48, 10853.

[34] Halter, D. P.; Heinemann, F. W.; Maron, L.; Meyer, K. Nat. Chem. 2017, 10, 259.

[35] Halter, D. P.; Heinemann, F. W.; Bachmann, J.; Meyer, K. Nature 2016, 530, 317.

[36] Bogart, J. A.; Lippincott, C. A.; Carroll, P. J.; Schelter, E. J. Angew. Chem. Int. Ed. 2015, 54, 8222.

[37] Fang, H.; Cole, B. E.; Qiao, Y.; Bogart, J. A.; Cheisson, T.; Manor, B. C.; Carroll, P. J.; Schelter, E. J. Angew. Chem. Int. Ed. 2017, 56, 13450.

[38] Solola, L. A.; Zabula, A. V.; Dorfner, W. L.; Manor, B. C.; Carroll, P. J.; Schelter, E. J. J. Am. Chem. Soc. 2016, 138, 6928.

[39] Cheisson, T.; Kersey, K. D.; Mahieu, N.; McSkimming, A.; Gau, M. R.; Carroll, P. J.; Schelter, E. J. J. Am. Chem. Soc. 2019, 141, 9185.

[40] Wu, Q.-Y.; Wang, C.-Z.; Lan, J.-H.; Chai, Z.-F.; Shi, W.-Q. Dalton Trans. 2020, 49, 15895.

[41] Liu, K.; Yu, J.-P.; Wu, Q.-Y.; Tao, X.-B.; Kong, X.-H.; Mei, L.; Hu, K.-Q.; Yuan, L.-Y.; Chai, Z.-F.; Shi, W.-Q. Organometallics 2020 , $39,4069$.

[42] Wu, Q.-Y.; Lan, J.-H.; Wang, C.-Z.; Zhao, Y.-L.; Chai, Z.-F.; Shi, W.-Q. J. Phys. Chem. A 2015, 119, 922.

[43] Wu, Q.-Y.; Wang, C.-Z.; Lan, J.-H.; Xiao, C.-L.; Wang, X.-K.; Zhao, Y.-L.; Chai, Z.-F.; Shi, W.-Q. Inorg. Chem. 2014, 53, 9607. 